\title{
The Influence of Biological Maturity on Dynamic Force-Time Variables and Vaulting Performance in Young Female Gymnasts
}

\author{
Sylvia Moeskops ${ }^{1}$. Jon L. Oliver ${ }^{1,2}$. Paul J. Read ${ }^{4,10} \cdot$ John B. Cronin ${ }^{2}$. Gregory D. Myer ${ }^{5,6,7} \cdot$ G. Gregory Haff ${ }^{8,9}$. \\ Isabel S. Moore ${ }^{1} \cdot$ Rhodri S. Lloyd ${ }^{1,2,3}$
}

Received: 27 April 2020 / Accepted: 28 June 2020 / Published online: 5 August 2020

(c) The Author(s) 2020

\begin{abstract}
Purpose This cross-sectional study investigated dynamic force-time variables and vaulting performance in young female gymnasts of different maturity status.

Methods 120 gymnasts aged 5-14 years were sub-divided into maturity groupings using percent of predicted adult height (\% PAH) attained. Participants performed three jumping protocols, the squat jump (SJ), countermovement jump (CMJ) and drop jump (DJ), before completing straight jump vaults that were recorded using two-dimensional video.

Results Jumping performance improved with biological maturity evidenced by the most mature gymnasts' producing significantly more absolute force $(P<0.05$; all $d>0.78)$, impulse $(P<0.05$; all $d>0.75)$ and power $(P<0.05$; all $d>0.91)$ than the least mature group, resulting in the greater jump heights $(P<0.05$; all $d>0.70)$. While, no significant differences were observed in relative peak force across multiple tests, measures of relative peak power did significantly increase with maturity. Based upon regression analyses, maturation was found to influence vertical take-off velocity during vaulting, explaining $41 \%$ of the variance in each jumping protocol. Across all tests, the DJ was found to have the highest predictive ability of vaulting vertical take-off velocity, explaining $55 \%$ of the total variance.

Conclusion Biological maturation impacts jump height and underpinning mechanical variables in young female gymnasts. Vaulting vertical take-off velocity appears to be influenced by maturation and various dynamic force-time variables, particularly those during DJ, which had the highest explained total variance.
\end{abstract}

Keywords Youth · Maturation · Squat jump · Countermovement jump · Drop jump · Gymnastics

Electronic supplementary material The online version of this article (https://doi.org/10.1007/s42978-020-00068-z) contains supplementary material, which is available to authorized users.

Sylvia Moeskops

smoeskops@cardiffmet.ac.uk

1 Youth Physical Development Centre, Cardiff School of Sport and Health Sciences, Cardiff Metropolitan University, Cyncoed Campus, Cyncoed Road, Cardiff, CF5 2YB, UK

2 Sports Performance Research Institute New Zealand (SPRINZ), AUT University, Auckland 1142, New Zealand

3 Centre for Sport Science and Human Performance, Waikato Institute of Technology, Hamilton 3240, New Zealand

4 Athlete Health and Performance Research Centre, Aspetar Orthopaedic and Sports Medicine Hospital, Doha, Qatar

5 Division of Sports Medicine, Cincinnati Children's Hospital, Cincinnati, OH, 45229, USA
6 Department of Pediatrics and Orthopaedic Surgery, College of Medicine, University of Cincinnati, Cincinnati, $\mathrm{OH} 45267$, USA

7 The Micheli Center for Sports Injury Prevention, Waltham, MA 02453, USA

8 Centre for Exercise and Sports Science, Edith Cowan University, Joondalup, WA 6027, Australia

9 Australian Centre for Research into Injury in Sport and Its Prevention (ACRISP), Edith Cowan University, Joondalup, WA 6027, Australia

10 University of Gloucestershire, Gloucester, GL50 2RH, UK 


\section{Introduction}

Jumping and rebounding are important prerequisites that underpin the high impact loading gymnastics skills (e.g. acrobatic series, tumbling etc.) [50]. Further, three of the four artistic disciplines that female gymnasts compete in (vault, beam and floor exercise) are heavily reliant on explosive lower-limb rebounding and jumping activities, which all utilize various expressions of the stretch-shortening cycle (SSC) [36]. Consequently, rebounding and jumping performance of artistic gymnasts are commonly assessed to identify key determinants of the sport $[8,29,31,50]$, determine physical profiles $[46,53]$ and evaluate the efficacy of training interventions $[7,11,28,37]$.

The mechanisms that underlie slow-SSC (ground contact time $>250 \mathrm{~ms}$ ) and fast-SSC (ground contact time $<250 \mathrm{~ms}$ ) may differ depending on the force-time characteristics of the movement [23] as well as the athlete's ability to perform efficient SSC mechanics [52]. For example, research indicates that the distribution and release of stored elastic energy is influenced by numerous factors including: the magnitude and rate of loading during the eccentric phase, stiffness and compliance of the muscle-tendon complex, and levels of pre-activation $[2,52]$. Researchers have emphasized the importance of measuring different expressions of SSC function in gymnasts as gymnastics skills involve both slow- and fast-SSC [37, 50]. Protocols that examine fastSSC function include drop jumps, repeated-hopping tasks and sprinting $[24,45]$, whereas slow-SSC tests typically involve countermovement jumps (CMJ) and standing long jumps [23]. Further, concentric only jumps which do not involve SSC function are frequently used as part of jumping test batteries [i.e. squat jump (SJ)] [4, 25, 50]. Comparisons of jump height or flight time between CMJ and SJ tests enables researchers to evaluate how effective gymnasts are at utilizing the contribution of the elastic energy during the braking phase $[4,29,50]$. However, despite the sport having high levels of early specialization, kinetic data in young female gymnasts is limited.

Previous age-related data comparing the jumping ability of female gymnasts aged 9-12 and 13-16 years has shown that jump height, maximal vertical force, as well as maximal and mean power all significantly increase with age [47]. Further, previous data has shown an increased age, a faster vault run-up speed and a shorter ground contact time during the handstand push off test, were important predictors of tumbling ability in female gymnasts aged 8-14 years [4]. Therefore, it appears that jumping performance in gymnasts increases naturally with age; however, assessing physical performance by chronological age as opposed to biological maturity does not account for large inter-individual variation in maturity status within a given age group [10]. Research shows maturation influences the development of physical qualities and motor skills in youth, particularly following the pubertal growth spurt [27]. For example, significant differences in absolute isometric peak force [34], vertical jump height [25] and sprint speed [32] have been reported between pre- and post-pubertal young athletes. As the timing and tempo of biological maturation differs between individuals of them same chronological age [27], analyzing testing data in young athletes according to maturity status has been recommended [21].

Existing gymnastics literature has often examined jump performance using field-based equipment such as contact mats [29, 30, 47], or methods which solely report performance outcomes such as jump height $[49,53]$. While these protocols provide surrogate measures of muscular power and SSC function in applied settings, superior insight can be gained from analyzing force-time data [38]. Specifically, this enables the identification of the mechanical variables that underpin jumping and rebounding performance, and ensures training prescription is more targeted to individual deficits. While some mechanistic $[4,35]$ and age-related jumping and rebounding data in young female gymnasts exists $[4,47]$, researchers have yet to examine such data in gymnasts grouped by different maturity status. Furthermore, the contribution of maturity and jumping force-time variables to vertical take-off velocity during vaulting performance is yet to be explored. Therefore, the first aim of this study was to examine the influence of maturity status on force-time variables from CMJ, SJ and drop jump (DJ) tests in young female gymnasts. The second aim of this study was to determine how these variables influence take-off velocity during vaulting performance.

\section{Methods}

\section{Participants}

One hundred and twenty female artistic gymnasts aged 5-14 years agreed to participate in the study. All participants had $>1$ years of gymnastics experience and were participating in gymnastics training 2-6 times per week, totaling 2-24 training hours per week. Participants were grouped according to biological maturity using percentage of predicted adult height (\%PAH) [14]: $<75 \% \mathrm{PAH}$, early prepubertal (early $\left.{ }_{\text {pre }} ; n=54\right) ; 76 \%-85 \% \mathrm{PAH}$, late pre-pubertal (late $\left._{\mathrm{pre}} ; n=47\right)$; and $86 \%-95 \% \mathrm{PAH}$, pubertal $(n=19)$. The groups were also matched by gymnastics-specific training hours per week $(\sim 11 \mathrm{~h} / \mathrm{w})$. Descriptive data for participants grouped by maturity status are shown in Table 1. Participants reported no injuries at the time of testing and were instructed to refrain from strenuous activity $24 \mathrm{~h}$ before testing. Written informed parental consent and participant 
Table 1 Descriptive statistics for all anthropometric variables (mean $\pm \mathrm{sd}$ )

\begin{tabular}{lccllllll}
\hline Group & $n$ & $\begin{array}{l}\text { Age } \\
\text { (years })\end{array}$ & $\begin{array}{l}\text { Standing height } \\
(\mathrm{cm})\end{array}$ & $\begin{array}{l}\text { Sitting height } \\
(\mathrm{cm})\end{array}$ & $\begin{array}{l}\text { Leg length } \\
(\mathrm{cm})\end{array}$ & $\begin{array}{l}\text { Body mass } \\
(\mathrm{kg})\end{array}$ & $\begin{array}{l}\text { Predicted \% } \\
\text { adult height }\end{array}$ & $\begin{array}{l}\text { Training hours } \\
\text { per week }\end{array}$ \\
\hline Early $_{\text {pre }}$ & 54 & $7.9 \pm 1.1$ & $124.5 \pm 8.8$ & $66.9 \pm 3.8$ & $57.7 \pm 5.5$ & $25.2 \pm 4.5$ & $70.1 \pm 4.0$ & $11.3 \pm 5.2$ \\
Late $_{\text {pre }}$ & 47 & $10.7 \pm 0.8^{\mathrm{a}}$ & $139.8 \pm 6.8^{\mathrm{a}}$ & $73.9 \pm 4.1^{\mathrm{a}}$ & $65.9 \pm 3.9^{\mathrm{a}}$ & $33.8 \pm 6.4^{\mathrm{a}}$ & $79.8 \pm 2.8^{\mathrm{a}}$ & $11.1 \pm 5.3$ \\
Pubertal & 19 & $12.8 \pm 0.8^{\mathrm{b}}$ & $150.4 \pm 5.6^{\mathrm{b}}$ & $78.2 \pm 2.7^{\mathrm{b}}$ & $72.3 \pm 2.7^{\mathrm{b}}$ & $45.1 \pm 9.5^{\mathrm{b}}$ & $89.2 \pm 3.2^{\mathrm{b}}$ & $11.0 \pm 6.1$ \\
\hline
\end{tabular}

Significant at the level of $P<0.05$

${ }^{\text {a }}$ Significantly greater than the early pre-pubertal group

${ }^{\mathrm{b}}$ Significantly greater than the early and late pre-pubertal groups

assent were obtained after ethical approval was granted by the local University Research Ethics Committee.

\section{Study Design}

This study used a cross-sectional design to examine jumping characteristics and vaulting performance in young artistic female gymnasts. All participants attended one testing session whereby anthropometric, SJ, CMJ, DJ and vaulting performance data were collected. Before testing commenced, participants performed a standardized 10-min dynamic warm-up led by the principal researcher, which included relevant activation and mobilization exercises, before advancing to one set of three SJ, CMJ and pogo hops. Familiarization of each testing protocol took place at the beginning of the testing session, which involved a demonstration and provision of standardized, child-friendly coaching cues. Participants then practiced the protocol until the principal investigator was satisfied with their technical competency.

\section{Anthropometrics}

Anthropometric data were collected, including standing and sitting height using a stadiometer to the nearest $0.1 \mathrm{~cm}$ (SECA, 321, Vogel \& Halke, Hamburg, Germany) and body mass using scales to the nearest $0.1 \mathrm{~kg}$ (SECA, 321, Vogel $\&$ Halke, Hamburg, Germany). Standing height (m), body mass $(\mathrm{kg})$, chronological age and parental height were used to determine participants' biological maturity status, using $\% \mathrm{PAH}[14]$.

\section{Jumping Protocols}

All jumping data were collected in a laboratory using two force plates sampling at a frequency of $1000 \mathrm{~Hz}$ (PASCO, 2 Axis force platforms, Roseville, CA 95747, USA). Participants were instructed to "stay as still as a statue" to optimize the stabilization of body weight during the first second of each test, before being given a countdown of "3, 2, 1 go". Gymnasts were instructed to keep their hands on their hips throughout and keep their legs extended during the flight phase of the jump. Three trials of each jumping protocol were completed with a minimum of $60 \mathrm{~s}$ passive rest between trials, to enable sufficient recovery [50]. All jumping data were filtered (MATLAB, R2018a or Labview LVRTE2014SP1; National Instruments) using a low-pass 4 th order recursive Butterworth filter. Based on residual analysis [54], the most appropriate cut-off frequency was found to be $13 \mathrm{~Hz}$. For the SJ and CMJ, the best trial selected for further analysis was determined by the highest jump. For the DJ, the best trial was determined by the highest springlike behavior correlation (i.e. a perfect inverse relationship is indicated by $r=-1.0$ ), which represents spring-mass model behavior [45]. All relative measures were calculated using body mass. Further information (abbreviations, units and descriptions) on the variables calculated from the SJ, CMJ and DJ tests can be found in Supplementary Tables 1-3.

\section{Squat Jump}

The SJ protocol required each participant to start in a semisquat position with approximately $90^{\circ}$ of knee flexion (determined subjectively by the rater) [26, 50]. Gymnasts were instructed to keep their hands on their hips and jump for maximum height after a countdown of " $3,2,1$ jump". Trials were discounted and repeated if the following occurred: a visible countermovement was present (either with the chest or lower limbs), hands did not remain on hips throughout the test, or if the lower limbs flexed during the flight phase. All SJ trials were analyzed by the same researcher using custom built analysis software (Labview, LVRTE2014SP1; National Instruments). Body weight was calculated by averaging the first second of force during the motionless period at the start of the jump when the participant was in the semi-squat position. Body weight plus 5 standard deviations (sd) was then used to identify the initiation of the jump [9]. Variables calculated included: jump height $(\mathrm{JH})$, peak velocity $\left(\mathrm{V}_{\text {peak }}\right)$, relative vertical impulse (Impulse rel $)$, absolute peak force $\left(\mathrm{PF}_{\mathrm{abs}}\right)$, relative peak force $\left(\mathrm{PF}_{\text {rel }}\right)$, absolute peak power $\left(\mathrm{PP}_{\mathrm{abs}}\right)$, relative peak power $\left(\mathrm{PP}_{\text {rel }}\right)$, absolute rate of force development $\left(\mathrm{RFD}_{\mathrm{abs}}\right)$ and relative rate of force development $\left(\mathrm{RFD}_{\text {rel }}\right)$. Using the highest RFD during a $20 \mathrm{~ms}$ 
time sampling window, absolute peak rate of force development $\left(\mathrm{pRFD}_{\mathrm{abs}}\right.$ ) and relative peak rate of force development $\left(\mathrm{pRFD}_{\text {rel }}\right)$ were also calculated.

\section{Countermovement Jump}

The CMJ protocol required each participant to squat to a self-selected knee, hip and ankle flexion angle and immediately jump for maximum height [50]. Trials were discounted and repeated if the gymnast's hands did not remain on their hips or, if their lower limbs flexed during the flight phase. All CMJ variables were calculated using a spreadsheet run through Microsoft Excel for Mac version 16.9 [5]. To identify the initiation of the jump, the first force value less than $5 \mathrm{sd}$ of body weight was used to increase the accuracy of the correct start point [5]. Furthermore, to optimize the accuracy of the velocity calculations (and in-turn the displacement and power calculations), the point of integration was identified as $-30 \mathrm{~ms}$ from the initiation of the gymnasts' jump, increasing the likelihood of the velocity being zero [5, 43]. To account for participant- or force plate-related noise, $5 \mathrm{sd}$ of $300 \mathrm{~ms}$ flight force was used to identify the take-off and landing threshold [5]. Variables calculated included: jump height $(\mathrm{JH})$, absolute peak force $\left(\mathrm{PF}_{\mathrm{abs}}\right)$, relative peak force $\left(\mathrm{PF}_{\text {rel }}\right)$, braking average impulse (Impulse brake $_{\text {e }}$ ), propulsive average impulse (Impulse ${ }_{\text {prop}}$ ), duration of braking phase $\left(\right.$ Time $\left._{\text {brake }}\right)$, duration of propulsive phase $\left(\right.$ Time $\left._{\text {prop }}\right)$, absolute peak power $\left(\mathrm{PP}_{\mathrm{abs}}\right)$, relative peak power $\left(\mathrm{PP}_{\text {rel }}\right)$, braking average power ( Power $_{\text {brake }}$ ) and propulsive average power (Power ${ }_{\text {prop}}$ ). It should be noted that braking phase starts at the end of the unweighting phase (when impulse drops below the bodyweight baseline) and ends when the athlete's velocity reaches zero or, when the impulse above baseline is equal to the impulse created during the unweighting phase [5]. Further, the propulsive phase occurs immediately after the braking phase and ends at the point of take-off and the athlete's has velocity has peaked just before "flight" [5].

\section{Drop Jump}

The DJ protocol required the participants to step out and off a $30 \mathrm{~cm}$ platform (positioned $10 \mathrm{~cm}$ from the contact area), land on two force plates, and rebound as high as possible with a fast ground contact time [45]. Participants were cued to "step out off of the box and rebound as high and as fast as possible" [45]. Trials where the gymnasts noticeably stepped down or jumped up from the platform were discounted and repeated. All DJ data were analyzed by the principal researcher using a custom-built Matlab (MATLAB, R2018a) analysis software. Variables calculated included: jump height $(\mathrm{JH})$, ground contact time (GCT), reactive strength index (RSI), centre of mass displacement $(\triangle \mathrm{COM})$, relative vertical leg stiffness $\left(\right.$ Stiffness $\left._{\text {rel }}\right)$ spring-like correlation (SLC), take-off velocity (TOV), braking average power (Power brake $_{\text {, }}$, propulsive average power (Power ${ }_{\text {prop }}$ ), braking average work (Work brake $_{\text {) and propulsive }}$ average work (Work prop $_{\text {) }}$ ).

\section{Vaulting}

Two-dimensional video analysis was used to determine the gymnasts' vertical take-off velocity $(\mathrm{m} / \mathrm{s})$ from the springboard during the execution of the straight vault. One stationary high-speed camera (Sony, RX10 mark 3) operating at $250 \mathrm{~Hz}$ and a shutter speed of $1 / 500$ of a second, was positioned $6 \mathrm{~m}$ perpendicular to the springboard where take-off occurred. The vaulting springboard was positioned $30 \mathrm{~cm}$ from the landing mat for all participants and adjusted after each trial to the same position using permanent floor markers. The approach run up distance was determined by the standard vaulting run-up distances for specific chronological age ranges; $10 \mathrm{~m}$ for $5-8$ years old, $12.5 \mathrm{~m}$ for $8-13$ years old and $15 \mathrm{~m}$ for $14-17$ years old. All gymnasts performed three straight jump vaults from a springboard (Continental, Fastlift Model) onto a landing mat (Continental, Safety Mat). The straight vault is the most basic of vaulting exercises and was chosen to ensure all gymnasts were capable of performing the skill regardless of competitive level or maturity status. An additional thin mat (Continental, Supplementary Soft-Landing Mat) which was shorter in length was placed on top of the landing mat, to encourage the gymnasts to perform the vault for maximum vertical jump height. All gymnasts received the standardized instruction "perform your highest straight jump to land on the thin mat." Trials were discounted and repeated if a participant; flexed their lower-limbs during the flight phase, fell forwards or backwards upon landing, or if they landed past the top mat. After each testing session, calibration was completed using a $4.0 \mathrm{~m}$ high calibration rod marked with $1 \mathrm{~m}$ intervals. All vaulting videos were analyzed using digitizing analysis software (Tracker v.5.0.5) by the principal researcher. Digitizing was performed using a marker that was placed on the gymnasts' greater trochanter at the time of testing to increase accuracy. Vaulting coordinate data were filtered (MATLAB, R2018a) using a low-pass 4th order recursive Butterworth filter. Based on residual analysis [54], the most appropriate cut-off frequency was found to be $10 \mathrm{~Hz}$. Vertical take-off velocity from the springboard was calculated using the Central Difference Method [54]. The best vault was determined as the highest straight jump (using the hip marker position) which was used for further analyses.

\section{Statistical Analyses}

Descriptive statistics (mean values \pm sd) were calculated for all variables from the jumping and vaulting data for each 
maturity group. Between-group differences in jumping and vaulting variables were assessed using a one-way analysis of variance (ANOVA). Homogeneity of variance was assessed via Levene's statistic, and where violated, Welch's adjustment was used to correct the $F$-ratio. Post-hoc analysis was used to identify the groups that were significantly different to one another using either Bonferroni or Games-Howell test, where equal variances were and were not assumed, respectively. Effect sizes (Cohen's $d$ ) were also calculated to establish the magnitude of any between-group differences [6] using the following classifications: $<0.2$ trivial; 0.2-0.59 small; 0.6-1.19 moderate; $1.2-2.0$ large; $2.0-4.0$ very large; $>4.0$ nearly perfect [13]. Pearson correlation coefficients were used to determine the strength of relationships between all jump test variables and vertical take-off velocity for the whole sample. The strength of these relationships was classified as either: $<0.2$ no relationship; $0.2-0.45$ weak; $0.46-0.7$ moderate; $>0.7$ strong, based on previous recommendations [41]. For each jump test, stepwise multiple regression analyses were employed separately to establish the contribution of jump variables and maturity status (\% PAH) on vertical take-off velocity from the spring board across the entire sample. The assumption of independent errors during the multiple regression analyses was tested via a series of Durbin-Watson tests, whilst multi-collinearity was tested using variance inflation factor (VIF) and tolerance diagnostics ( 0.2 tolerance cut-off). All significance values were accepted at $P<0.05$ and all statistical procedures were conducted using SPSS v.24 for Macintosh.

\section{Results}

\section{Squat Jump}

Data showed small to moderate, non-significant betweengroup differences for JH $(P>0.05$; Fig. 1$)$. Results for all other SJ variables are presented in Table 2. Small to moderate significant increases in $\mathrm{V}_{\text {peak }}$, Impulse $\mathrm{rel}_{\text {rel }}, \mathrm{PP}_{\text {abs }}$ and $\mathrm{PP}_{\text {rel }}$ between the early $y_{\text {pre }}$ and pubertal groups and between

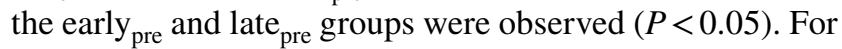
$\mathrm{PF}_{\mathrm{abs}}$, there was a moderate significant increase between the early $y_{\text {pre }}$ and pubertal and late pre $_{\text {groups }}(P<0.05)$. No significant differences were indicated between any of the groups for $\mathrm{PF}_{\text {rel }}$ and all effect sizes were trivial. $R F D_{a b s}$ showed small-moderate significant increases between the early $_{\text {pre }}$ and pubertal groups $(P<0.05)$ and late pre $_{\text {groups }}$ $(P<0.05)$. Between-group differences for all other RFD variables $\left(\mathrm{RFD}_{\text {rel }}, \mathrm{pRFD}_{\mathrm{abs}}\right.$ and $\mathrm{pRFD}$ rel $)$ were all found to be non-significant and trivial or small. No significant differences were found between the late ${ }_{\text {pre }}$ and pubertal for any variables, and all effect sizes were trivial to small.

\section{Countermovement Jump}

Moderate significant increases in JH were found between the early $\mathrm{pre}_{\text {and pubertal groups }(P<0.05 \text {; Fig. } 1) \text { and late }}$ pre groups $(P<0.05$; Fig. 1$)$. Results for all other CMJ variables are presented in Table 3. Moderate to large significant increases were present between the pubertal group and both the early ${ }_{\text {pre }}$ and late ${ }_{\text {pre }}$ groups for $\mathrm{PF}_{\mathrm{abs}}$, Impulse $_{\mathrm{Brake}}$, Impulse $_{\text {Prop, }} \mathrm{PP}_{\mathrm{abs}}$, Power $_{\text {brake }}$ and Power Prop $(P<0.05)$. For these variables, moderate to large increases were also found between the early pre $_{\text {and late }}$ pre groups $(P<0.05)$. Significant moderate increases in $\mathrm{PP}_{\text {rel }}$ were present between the early $y_{\text {pre }}$ and late $e_{\text {pre }}$ and between the early pre $_{\text {and pubertal groups }}$ $(P<0.05)$. Non-significant, trivial to small between-group differences were reported for $\mathrm{PF}_{\text {rel }}$, Time $_{\text {Brake }}$ and Time ${ }_{\text {Prop }}$ $(P>0.05)$.

\section{Drop Jump Results}

Moderate significant increases in JH were shown between the early ${ }_{\text {pre }}$ and pubertal groups $(P<0.05$; Fig. 1$)$; while, small significant increases were found between the early pre $_{\text {p }}$ and late ${ }_{\text {pre }}$ groups $(P<0.05$; Fig. 1$)$. The remaining DJ
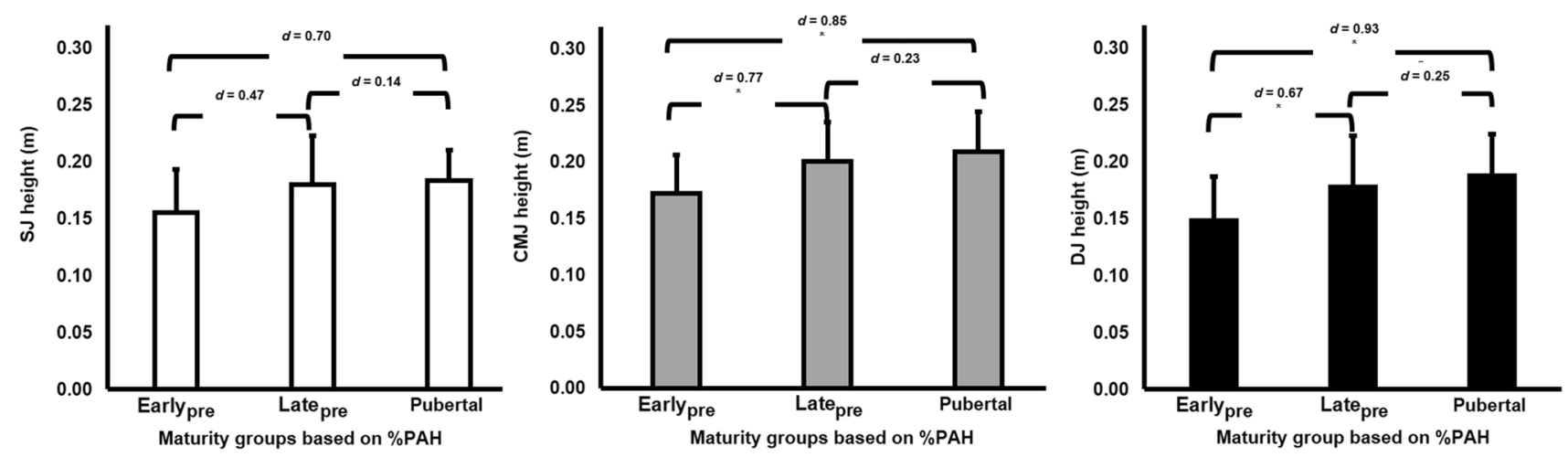

Fig. 1 Maturity group analysis of jump height (m) from the squat jump, countermovement jump and drop jump tests respectively (mean \pm sd) 
Table 2 Maturity group analysis of variables from the squat jump test (mean \pm sd)

\begin{tabular}{|c|c|c|c|c|c|c|}
\hline \multirow[t]{2}{*}{ Test variable } & \multirow[t]{2}{*}{ Early $_{\text {pre }}$} & \multirow[t]{2}{*}{ Late $_{\text {pre }}$} & \multirow[t]{2}{*}{ Pubertal } & \multicolumn{3}{|c|}{ Between group effect size $(d)$} \\
\hline & & & & Early $_{\text {pre }}-$ Late $_{\text {pre }}$ & Late $_{\text {pre }}-$ Pubertal & $\begin{array}{l}\text { Early }_{\text {pre }}- \\
\text { Pubertal }\end{array}$ \\
\hline $\mathrm{V}_{\text {peak }}(\mathrm{m} / \mathrm{s})$ & $1.97 \pm 0.21$ & $2.12 \pm 0.17^{a}$ & $2.14 \pm 0.12^{a}$ & 0.53 & 0.05 & 0.55 \\
\hline $\operatorname{Impulse}_{\text {rel }}(\mathrm{m} \cdot \mathrm{s})$ & $1.72 \pm 0.27$ & $1.85 \pm 0.34^{a}$ & $1.98 \pm 0.15^{a}$ & 0.37 & 0.34 & 0.75 \\
\hline $\mathrm{PF}_{\mathrm{abs}}(\mathrm{N})$ & $591.14 \pm 206.91$ & $756.25 \pm 174.99^{a}$ & $793.18 \pm 208.40^{a}$ & 0.76 & 0.18 & 0.84 \\
\hline $\mathrm{PF}_{\text {rel }}(\mathrm{N} / \mathrm{kg})$ & $21.77 \pm 3.41$ & $21.54 \pm 2.42$ & $21.74 \pm 1.40$ & 0.06 & 0.06 & 0.02 \\
\hline $\mathrm{PP}_{\mathrm{abs}}(\mathrm{W})$ & $933.37 \pm 1302.96$ & $1302.96 \pm 387.24^{a}$ & $1360.64 \pm 479.61^{a}$ & 0.89 & 0.09 & 0.91 \\
\hline $\mathrm{PP}_{\text {rel }}(\mathrm{W} / \mathrm{kg})$ & $33.39 \pm 5.71$ & $36.65 \pm 4.42^{a}$ & $37.44 \pm 3.24^{a}$ & 0.48 & 0.13 & 0.60 \\
\hline $\mathrm{RFD}_{\mathrm{abs}}(\mathrm{N} / \mathrm{s})$ & $1160.20 \pm 499.91$ & $1457.19 \pm 518.07^{a}$ & $1571.72 \pm 549.38^{a}$ & 0.55 & 0.21 & 0.68 \\
\hline $\mathrm{RFD}_{\text {rel }}(\mathrm{N} / \mathrm{kg} / \mathrm{s})$ & $43.81 \pm 18.09$ & $42.14 \pm 14.05$ & $43.51 \pm 13.11$ & 0.10 & 0.09 & 0.05 \\
\hline $\mathrm{pRFD}_{\mathrm{abs}}(\mathrm{N} / \mathrm{s})$ & $3691.94 \pm 4264.53$ & $4069.38 \pm 4303.35$ & $3710.09 \pm 1905.21$ & 0.09 & 0.10 & 0.02 \\
\hline $\mathrm{pRFD}_{\text {rel }}(\mathrm{N} / \mathrm{kg} / \mathrm{s})$ & $135.56 \pm 132.10$ & $119.41 \pm 138.07$ & $102.98 \pm 46.63$ & 0.12 & 0.14 & 0.26 \\
\hline
\end{tabular}

Significant at the level of $P<0.05$

${ }^{a}$ Significantly greater than the early pre-pubertal group

$V_{\text {peak }}$ peak velocity, Impulse $e_{r e l}$ relative vertical net impulse, $P F_{a b s}$ absolute peak force, $P F_{\text {rel }}$ relative peak force, $P P_{a b s}$ absolute peak power, $P P_{r e l}$ relative peak power, $R F D_{a b s}$ absolute rate of force development, $R F D_{r e l}$ relative rate of force development, $p R F D$ absolute peak rate of force development

Small effect size (0.20-0.59); Moderate effect size (0.60-1.19); Large effect size (1.20-2.00)

Table 3 Maturity group analysis of variables from the countermovement jump test (mean $\pm \mathrm{sd}$ )

\begin{tabular}{|c|c|c|c|c|c|c|}
\hline \multirow[t]{2}{*}{ Test variable } & \multirow[t]{2}{*}{ Early $_{\text {pre }}$} & \multirow[t]{2}{*}{ Late $_{\text {pre }}$} & \multirow[t]{2}{*}{ Pubertal } & \multicolumn{3}{|c|}{ Between group effect size $(d)$} \\
\hline & & & & Early $_{\text {pre }}-$ Late $_{\text {pre }}$ & Late $_{\text {pre }}-$ Pubertal & $\begin{array}{l}\text { Early }_{\text {pre }} \\
\text { Pubertal }\end{array}$ \\
\hline $\mathrm{PF}_{\mathrm{abs}}(\mathrm{N})$ & $350.84 \pm 115.05$ & $508.94 \pm 156.42^{a}$ & $607.86 \pm 111.55^{b}$ & 1.01 & 0.66 & 1.52 \\
\hline $\mathrm{PF}_{\text {rel }}(\mathrm{N} / \mathrm{kg})$ & $13.95 \pm 3.72$ & $14.87 \pm 3.08$ & $14.11 \pm 3.25$ & 0.27 & 0.25 & 0.04 \\
\hline $\operatorname{Impulse}_{\text {brake }}(\mathrm{N} \cdot \mathrm{s})$ & $22.07 \pm 9.27$ & $32.63 \pm 9.03^{a}$ & $46.14 \pm 9.17^{b}$ & 1.00 & 1.24 & 1.67 \\
\hline Time $_{\text {brake }}(\mathrm{s})$ & $0.373 \pm 0.187$ & $0.457 \pm 0.434$ & $0.358 \pm 0.205$ & 0.26 & 0.26 & 0.07 \\
\hline $\operatorname{Impulse}_{\text {prop }}(\mathrm{N} \cdot \mathrm{s})$ & $46.16 \pm 10.83$ & $68.32 \pm 15.53^{a}$ & $88.45 \pm 14.29^{b}$ & 1.29 & 1.14 & 1.82 \\
\hline Time $_{\text {prop }}(\mathrm{s})$ & $0.248 \pm 0.068$ & $0.246 \pm 0.053$ & $0.253 \pm 0.062$ & 0.03 & 0.13 & 0.07 \\
\hline $\mathrm{PP}_{\mathrm{abs}}(\mathrm{W})$ & $894.37 \pm 234.39$ & $1343.09 \pm 337.62^{a}$ & $1756.29 \pm 303.03^{b}$ & 1.23 & 1.14 & 1.85 \\
\hline $\mathrm{PP}_{\text {rel }}(\mathrm{W} / \mathrm{kg})$ & $35.35 \pm 5.01$ & $39.23 \pm 4.97^{a}$ & $40.35 \pm 4.95^{a}$ & 0.73 & 0.23 & 0.73 \\
\hline Power $_{\text {brake }}(\mathrm{W})$ & $-99.12 \pm 40.14$ & $-135.32 \pm 54.66^{a}$ & $-200.12 \pm 63.63^{b}$ & 0.72 & 1.01 & 1.51 \\
\hline Power $_{\text {prop }}(\mathrm{W})$ & $490.71 \pm 148.27$ & $726.78 \pm 200.30^{a}$ & $947.21 \pm 117.43^{b}$ & 1.12 & 1.07 & 1.77 \\
\hline
\end{tabular}

Significant at the level of $P<0.05$

${ }^{a}$ Significantly greater than the early pre-pubertal group

${ }^{b}$ Significantly greater than the early pre-pubertal, and the late pre-pubertal groups

$P F_{a b s}$ absolute peak force, $P F_{\text {rel }}$ relative peak force, Impulse brake $_{\text {braking impulse, Time }}$ brake braking phase duration, Impulse $e_{\text {prop }}$ propulsive impulse, Time $e_{\text {prop }}$ propulsive phase duration, $P P_{a b s}$ absolute peak power, $P P_{\text {rel }}$ relative peak power, $P$ ower $r_{\text {brake }}$ braking average power, Powerprop propulsive average power

Small effect size (0.20-0.59); Moderate effect size (0.60-1.19); Large effect size (1.20-2.00)

variables are displayed in Table 4. Moderate significant increases in stiffness $s_{\text {rel }}$ were found between the early pre $_{\text {p }}$ and pubertal groups only $(P<0.05)$. For $\triangle \mathrm{COM}$, a small, significant increase was present between the early $y_{\text {pre }}$ and pubertal groups $(P<0.05)$ as well as the early ${ }_{\text {pre }}$ and late $_{\text {pre }}$ groups $(P<0.05)$. Large, significant increases in Power $_{\text {brake }}$, Power ${ }_{\text {prop }}$, Work brake $_{\text {and }}$ Work prop $_{\text {were found }}$ between the early pre and pubertal groups $(P<0.05)$ and 
Table 4 Maturity group analysis of variables from the drop jump test (mean \pm sd)

\begin{tabular}{|c|c|c|c|c|c|c|}
\hline \multirow[t]{2}{*}{ Test variable } & \multirow[t]{2}{*}{ Early $_{\text {pre }}$} & \multirow[t]{2}{*}{ Late $_{\text {pre }}$} & \multirow[t]{2}{*}{ Pubertal } & \multicolumn{3}{|c|}{ Between group effect size $(d)$} \\
\hline & & & & Early $_{\text {pre }}-$ Late $_{\text {pre }}$ & Late $_{\text {pre }}-$ Pubertal & $\begin{array}{l}\text { Early }_{\text {pre }}- \\
\text { Pubertal }\end{array}$ \\
\hline GCT (s) & $0.193 \pm 0.049$ & $0.191 \pm 0.340$ & $0.214 \pm 0.077$ & 0.10 & 0.32 & 0.29 \\
\hline RSI & $0.80 \pm 0.26$ & $0.95 \pm 0.29$ & $0.96 \pm 0.32$ & 0.43 & 0.10 & 0.58 \\
\hline $\mathrm{PF}(\mathrm{N})$ & $1549.00 \pm 382.65$ & $2070.72 \pm 472.30^{a}$ & $1918.52 \pm 629.35$ & 1.04 & 0.29 & 0.78 \\
\hline$\Delta \mathrm{COM}(\mathrm{cm})$ & $9.91 \pm 2.67$ & $11.34 \pm 2.58^{a}$ & $12.11 \pm 5.40^{a}$ & 0.59 & 0.01 & 0.53 \\
\hline Stiffness $_{\text {rel }}(B W / m)$ & $14.72 \pm 4.58$ & $17.54 \pm 5.16$ & $24.27 \pm 18.27^{a}$ & 0.18 & 0.62 & 0.72 \\
\hline SLC & $-0.92 \pm 0.05$ & $-0.94 \pm 0.05$ & $-0.94 \pm 0.05$ & 0.27 & 0.14 & 0.27 \\
\hline TOV (m/s) & $1.91 \pm 0.19$ & $2.00 \pm 0.17$ & $1.84 \pm 0.37$ & 0.24 & 0.66 & 0.25 \\
\hline Power $_{\text {brake }}(\mathrm{W})$ & $-901.10 \pm 199.90$ & $-1278.17 \pm 285.17^{a}$ & $-1402.65 \pm 372.30^{a}$ & 0.91 & 0.43 & 1.21 \\
\hline Power $_{\text {prop }}(\mathrm{W})$ & $749.40 \pm 176.02$ & $1116.34 \pm 259.12^{a}$ & $1252.18 \pm 266.50^{a}$ & 1.12 & 0.49 & 1.45 \\
\hline Work $_{\text {brake }}(\mathrm{J})$ & $66.35 \pm 17.42$ & $103.60 \pm 29.83^{a}$ & $125.59 \pm 38.23^{a}$ & 1.14 & 0.50 & 1.51 \\
\hline Work $_{\text {prop }}(\mathrm{J})$ & $46.35 \pm 16.45$ & $79.63 \pm 29.20^{a}$ & $102.00 \pm 50.50^{a}$ & 1.17 & 0.43 & 1.40 \\
\hline
\end{tabular}

Significant at the level of $P<0.05$

${ }^{a}$ Significantly greater than the early pre-pubertal group

GCT ground contact time, RSI reactive strength index, $\triangle C O M$ centre of mass displacement, SLC spring-like correlation, TOV take-off velocity, $P F_{a b s}$ absolute peak force, Power $_{\text {brake }}$ braking average power, Power $_{\text {prop }}$ propulsive average power, Work brake $_{\text {braking average work, Work }}$ prop propulsive average work, Stiffness rel $_{\text {relative vertical stiffness }}$

Small effect size (0.20-0.59); Moderate effect size (0.60-1.19); Large effect size (1.20-2.00)

moderate, significant increases between the early ${ }_{\text {pre }}$ and late $_{\text {pre }}$ groups $(P<0.05)$. No significant differences were found between any groups for GCT, RSI, SLC and TOV and effect sizes ranged from trivial to moderate. Differences for all DJ variables between the late pre $_{\text {and }}$ pubertal groups were non-significant and trivial to moderate.

\section{Vaulting}

Moderate, significant increases in vaulting vertical takeoff velocity were found between the early ${ }_{\text {pre }}$ and late ${ }_{\text {pre }}$ groups $(P<0.05)$ and between the early ${ }_{\text {pre }}$ and pubertal groups $(P<0.05)$. However, no significant differences were observed between the late ${ }_{\text {pre }}$ and pubertal groups for vertical take-off velocity and effect sizes were trivial.

\section{Regression Analyses}

Multiple stepwise regression analysis outputs for each jumping test across the whole sample is shown in Table 5. For the SJ test, regression analysis showed that variation in vertical take-off velocity during vaulting performance was best

Table 5 Stepwise multiple linear regression equations explaining the variables that significantly $(P<0.05)$ contributed to vertical take-off velocity during vaulting from the SJ, CMJ and DJ tests for all maturity groups

\begin{tabular}{|c|c|c|c|c|c|}
\hline $\begin{array}{l}\text { Jumping pro- } \\
\text { tocol }\end{array}$ & Dependent variable & $\begin{array}{l}\text { Independent vari- } \\
\text { ables }\end{array}$ & $\begin{array}{l}\text { Regression equation (beta } \\
\text { coefficients) }\end{array}$ & $\begin{array}{l}\text { Adjusted } R^{2} \\
\text { value }\end{array}$ & Sig. value \\
\hline \multirow[t]{3}{*}{ SJ } & \multirow[t]{3}{*}{ Vertical take-off velocity from springboard } & Constant & -0.787 & & \\
\hline & & $\% \mathrm{PAH}$ & 0.044 & 0.406 & 0.000 \\
\hline & & $\mathrm{PP}_{\mathrm{abs}}$ & 0.000 & 0.454 & 0.003 \\
\hline \multirow[t]{3}{*}{ CMJ } & \multirow[t]{3}{*}{ Vertical take-off velocity from springboard } & Constant & -1.248 & & \\
\hline & & $\% \mathrm{PAH}$ & 0.046 & 0.406 & 0.000 \\
\hline & & $\mathrm{JH}$ & 3.761 & 0.435 & 0.008 \\
\hline \multirow[t]{4}{*}{ DJ } & \multirow[t]{4}{*}{ Vertical take-off velocity from springboard } & Constant & -0.165 & & \\
\hline & & $\% \mathrm{PAH}$ & 0.053 & 0.406 & 0.000 \\
\hline & & GCT & -0.008 & 0.514 & 0.000 \\
\hline & & $\triangle \mathrm{COM}$ & 0.067 & 0.548 & 0.002 \\
\hline
\end{tabular}

$P P_{a b s}$ absolute peak power, $\% P A H$ percent of predicted adult height attained, $J H$ jump height, $G C T$ ground contact time, $\triangle C O M$ centre of mass displacement 
explained by $\% \mathrm{PAH}(41 \%)$ and greater $\mathrm{PP}_{\mathrm{abs}}(4 \%)$, accounting for $45 \%$ of the total variance. While $\% \mathrm{PAH}(41 \%)$ and higher $\mathrm{JH}(3 \%)$ were the best predictors from the CMJ test, explaining $44 \%$ of the total variance. Finally, the DJ test was found to have highest explained total variance (55\%) and was best explained by \%PAH (41\%), reduced GCT (10\%) and greater $\triangle \mathrm{COM}(4 \%)$.

\section{Discussion}

This study examined the influence of maturity status on force-time variables from CMJ, SJ and DJ tests and the influence of these variables on vaulting performance in young female gymnasts. Overall, the main findings of this study were that jumping performance (i.e. jump height being the outcome measure) improves with biological maturity. This was evidenced by the most mature gymnasts' producing significantly more impulse ${ }_{\text {prop}}$, power (both peak and average power) and faster $\mathrm{V}_{\text {peak }}$ than the least mature group, resulting in the greater jump heights in all jump tests. While, no significant differences were observed in relative peak force across multiple tests, measures of relative peak power did significantly increase. Jumping variables across the different tests explained only a small amount of the variance in vertical take-off velocity during vaulting which appeared to be more strongly associated with \% $\mathrm{PAH}$, indicating its potential role in vaulting performance.

Small and moderate increases in $\mathrm{JH}$, albeit non-significant, were reported between the least mature group and the late $e_{\text {pre }}$ and pubertal groups for the SJ testing. Our findings are consistent with previous SJ data, which found no significant difference in jump height between under-11 s and under-13 s (both groups were pre-peak height velocity (PHV)), albeit in male youth soccer players [25]. In contrast, SJ jump height was significantly greater between under-16 $\mathrm{s}$ (post-PHV) and both less mature groups of boys [25]. With further growth and maturation, post-pubertal female gymnasts could produce greater amounts of force, impulse and power, resulting in significantly higher jump heights than less mature girls. However, the natural increases in fat-mass females experience with biological maturation could negatively impact jumping height [27].

The observed increases in jump height can be explained by the significant increases in Impulse ${ }_{\text {rel }}$ and $V_{\text {peak }}$ young gymnasts experience with maturity. Impulse rel $_{\text {provides }}$ insight into athletes' velocity capacity, which directly influences vertical jumping performance [15, 51]. Further, significant increases in $\mathrm{PF}_{\mathrm{abs}}, \mathrm{PP}_{\mathrm{abs}}$, and $\mathrm{RFD}_{\mathrm{abs}}$ were evident between the least mature group of gymnasts and both late ${ }_{\text {pre }}$ and pubertal groups. These results are likely due to the maturity-associated increases in force-producing capabilities that occur as children approach adolescence [48]. However, when normalized to body mass, only Impulse $e_{\text {rel }}$ and $\mathrm{PP}_{\text {rel }}$ significantly increased with maturity between the early ${ }_{\text {pre }}$ and more mature groups, while all other relative measures $\left(\mathrm{PF}_{\text {rel }}, \mathrm{RFD}_{\text {rel }}\right.$ and $\left.\mathrm{pRFD} \mathrm{rel}_{\text {rel }}\right)$ remained unchanged. This finding corroborates with existing age-related SJ literature, which has shown a significant age effect for $\mathrm{PP}_{\text {rel }}$ but not $\mathrm{PF}_{\text {rel }}$ in young female gymnasts [4]. Given that the amount of relative force produced appears stable with advancing maturity, these data could indicate that maturity-related increases in SJ height may be attributed to faster movement velocities as evidenced by the difference in $\mathrm{PP}_{\text {rel }}$ and $\mathrm{V}_{\text {peak }}$. Specifically, these increases in movement velocity appear to be due to greater changes in contraction distance which, might be driven by growth (i.e. longer levers and fascicle lengths) and jumping strategy (i.e. taller, more mature gymnasts move a greater distance to get to a similar optimal depth prior to push-off) [1, 48].

Small to moderate significant increases in CMJ height between successive maturity groups was found in this study. These results support previous researchers who have shown CMJ height increases with advancing age and maturity throughout childhood and adolescence [12, 19, 22, 27]. While data from the present study aligns with existing literature, less is known about the underlying kinetics. Moderate to large increases were reported in absolute kinetic variables $\left(\mathrm{PF}_{\mathrm{abs}}\right.$, Impulse $_{\text {brake }}$, Impulse $_{\text {prop }}, \mathrm{PP}_{\mathrm{abs}}$, Power $_{\text {brake }}$ and Power ${ }_{\text {prop }}$ ) between successive groups. It is therefore likely that the significantly greater impulse more mature gymnasts produced resulted in higher jump heights, than their immature counterparts. This is further evidenced by the moderate to large significant increases in Impulse brake $_{\text {and Impulse }}$ prop gymnasts experience with increasing maturity while, the duration of these phases remains unchanged.

For $\mathrm{PF}_{\text {rel }}$ and $\mathrm{PP}_{\text {rel }}$ a similar pattern to the results from the SJ was observed, with no significant differences between any groups for $\mathrm{PF}_{\text {rel }}$ and only a significant increase in $\mathrm{PP}_{\text {rel }}$ from the least mature gymnasts to the late $\mathrm{pre}_{\text {re }}$ and pubertal groups, respectively. Previous data in young female gymnasts has also shown $\mathrm{PF}_{\text {rel }}$ is unchanged with maturation during this period of development, albeit during an isometric mid-thigh pull protocol [35]. Together, these results suggest young female gymnasts could benefit from strength and conditioning that offers an alternative training stimulus to enhance relative strength and movement velocity, beyond that of sport-specific training.

Maturation appears to enhance young gymnasts' ability to rebound higher during the DJ protocol, evidenced by moderate, significant increases in jump height between the early pre $_{\text {group and both late }}$ pre and pubertal cohorts of gymnasts. The significantly greater amount of PF, work, power and stiffness $s_{\text {rel }}$ more mature gymnasts produce, likely explains their superior ability to jump higher than their more immature peers. All maturity groups were able to meet the 
required GCT $<250 \mathrm{~ms}$ for fast-SSC function which is noteworthy, and may reflect selection and/or training effect of gymnastics in this population. Fast-SSC actions are thought to promote greater movement speed via mechanisms inclusive of; elastic energy reutilization, greater pre-activation, stretch-reflex contributions and greater neural excitation [3, $16,20,48]$. Thus, maturity-related increases in kinetic variables in this study are likely attributed to structural and neural adaptations [48]. Specifically, natural increases in tendon CSA and stiffness [17, 40], increases in preactivation [18, 42], reduced co-contraction ratios [18] and so forth, may enhance SSC function in youth. However, it should be noted that no significant differences between the two most mature groups for jump height, or any other DJ variables were detected which, could be due to the significant increases in \% PAH and body mass in the more mature cohort.

The results for RSI and SLC revealed no significant differences between all maturity groups, although some small increases with advancing maturity were present. Specifically, the trend of increasing RSI with maturation appears to be driven by primarily increases in jump height as no significant differences in GCT were observed. While RSI can increase through a potentially undesirable strategy (i.e. as it is a ratio determined by JH and GCT), the inclusion of the SLC allows further evaluation of athletes' SSC capabilities [45]. Current research suggests that spring-like behavior is represented by a SLC of above 0.8 , whereby effective SSC mechanisms facilitate storage and reutilization of elastic energy within connective tissues [44]. Importantly, data from this study shows that all three cohorts of gymnasts display good spring-like behavior ( $>0.9$ ), and this remains stable throughout the development period examined.

\section{Regression Analyses}

Based upon our data it appears that maturation most strongly influences vertical take-off velocity during vaulting, evidenced by \% PAH appearing in all regression equations and explaining $\sim 41 \%$ of variance in each jumping test. Further, regression analysis revealed only one other variable predicted vertical take-off velocity during vaulting performance from the SJ and CMJ tests, $\mathrm{PP}_{\mathrm{abs}}(4 \%)$ and $\mathrm{JH}(3 \%)$ respectively. However, for the DJ protocol both a shorter GCT $(10 \%)$ and greater $\triangle \mathrm{COM}(4 \%)$ were identified as predictors. Together with \% $\mathrm{PAH}$, these variables explained 55\% of common variance in vertical take-off velocity, resulting in the DJ test explaining the most variance in the vault straight jump. These results are perhaps unsurprising given the similarities between the gymnasts' interaction with spring-board during take-off and the drop jump protocol, albeit on different types of surfaces. From a dynamic correspondence perspective, both require fast-SSC function owing to the constrained amount of time in contact with the ground or spring-board [39, 45]. These results highlight the importance of maturation and the ability to produce high amounts of force at faster rates for successful vaulting performance in young female gymnasts.

One limitation of this study is that the between group differences reported for the maturity groups were identified from a cross-sectional data set. Therefore, future research is required to track the natural development of youth female gymnasts across a longitudinal timeframe (i.e. from pre- to post-puberty) to confirm this study's findings. While the authors recognize this limitation, the current study makes a significant and novel contribution to the pediatric (and gymnastics) literature by examining differences in jump kinetics during jumping and vaulting, which can be used to help inform training prescription.

\section{Conclusion}

This study shows the value of using a jumping test battery that includes underpinning mechanical variables in young female gymnasts at different stages of maturation. Many absolute kinetic variables appear to significantly increase with advancing maturity across multiple tests however, we observed no differences in relative peak force while relative power and velocity significantly increased. Further, no significant differences were observed between maturity groups in braking and propulsive phase times for the CMJ test, or GCT for the DJ. Overall, this suggests more mature gymnasts have a higher movement velocity due to greater contraction distances over similar amounts of time. Therefore, as relative measures of strength do not appear to naturally increase with maturation, strength and conditioning provision for youth female gymnasts should target this physical quality throughout childhood and adolescence. This finding supports previous gymnastics-based literature which has demonstrated the effectiveness of resistance training interventions to increase levels of muscular strength and consequently, jumping performance $[28,33]$. Providing technical competency is maintained, long-term training programs should aim to provide gymnasts with an effective training stimulus that differs to their sports-specific training in an integrative and individual manner (e.g. using higher loading schemes via resistance training, weightlifting derivatives etc.).

As this study has shown biological maturation influences vertical take-off velocity during vaulting, practitioners should monitor and consider maturational status in testing batteries for youth gymnasts. Further, greater absolute peak power during the SJ, higher CMJ height and shorter GCTs and greater $\triangle \mathrm{COM}$ during the DJ, appear to be the most important variables for vaulting performance in the jumping tests examined. Targeting performance improvements 
in these measures within the training programs of young gymnasts seems logical. However, it is crucial that training programs are always developed holistically and must be inclusive of exercises which enhance gymnasts' overall athleticism and reduce the relative risk of gymnastics-related injuries.

Funding None.

\section{Compliance with Ethical Standards}

Conflict of interest The authors declare that they have no conflict of interest.

Ethics Approval Ethical approval was granted from Cardiff Metropolitan University's Ethics board.

Consent to Participate Written (signed) informed consent and assent was attained from the parents and children who participated in the study.

Consent for Publication This was included in the informed consent and assent forms and was attained from all parents and children who participated in the study.

Open Access This article is licensed under a Creative Commons Attribution 4.0 International License, which permits use, sharing, adaptation, distribution and reproduction in any medium or format, as long as you give appropriate credit to the original author(s) and the source, provide a link to the Creative Commons licence, and indicate if changes were made. The images or other third party material in this article are included in the article's Creative Commons licence, unless indicated otherwise in a credit line to the material. If material is not included in the article's Creative Commons licence and your intended use is not permitted by statutory regulation or exceeds the permitted use, you will need to obtain permission directly from the copyright holder. To view a copy of this licence, visit http://creativecommons.org/licenses/by/4.0/.

\section{References}

1. Asai H, Aoki J. Force development of dynamic and static contractions in children and adults. Int J Sports Med. 1996;17(3):170-4. https://doi.org/10.1055/s-2007-972827.

2. Blazevich A. The stretch-shortening cycle (SSC). In: Caedinale M, Newton R, Nosaka K. Strength and conditioning: biological principles and practical applications. 1st ed. Wiley-Blackwell, Oxford; 2011.

3. Bosco C, Montanari G, Tarkka I, Latteri F, Cozzi M, Iachelli G, Faina M, Colli R, Monte AD, Rosa ML, Ribacchi R, Giovenali P, Cortili G, Saibeness. The effect of pre-stretch on mechanical efficiency of human skeletal muscle. Acta Physiol Scand. 1987;131(3):323-9.

4. Bradshaw E, Le Rossignol P. Anthropometric and biomechanical field measures of floor and vault ability in 8 to 14 year old talentselected gymnasts. Sports Biomech. 2004;3(2):249-62. https:// doi.org/10.1080/14763140408522844.

5. Chavda S, Bromley T, Jarvis P, Williams S, Bishop C, Turner AN, Lake J, Mundy P. Force-Time characteristics of the countermovement jump: analyzing the curve in excel. J Strength Cond. 2018;40(2):66-77.
6. Cohen J. Statistical power analysis for the behavioral sciences. 2nd ed. New Jersey: Lawrence Erlbaum; 1988.

7. Colclough A, Munro AG, Herrington LC, McMahon JJ, Comfort P. The effects of a four week jump-training program on frontal plane projection angle in female gymnasts. Phys Ther Sport. 2018;30:29-33. https://doi.org/10.1016/j.ptsp.2017.11.003.

8. Dallas G, Zacharogiannis E, Paradisis G. Physiological profile of elite Greek gymnasts. J Phys Ed Sport. 2013;13(1):27-322. https ://doi.org/10.7752/jpes.2013.01005.

9. Dos'Santos T, Jones PA, Comfort P, Thomas C. Effect of different onset thresholds on isometric mid-thigh pull force-time variables. J Strength Cond Res. 2017;31(12):3463-73. https:// doi.org/10.1519/JSC.0000000000001765.

10. Faigenbaum A, Lloyd R, Oliver JL. Essentials of youth fitness. 1st ed. Champaign: Human Kinetics; 2020.

11. Hall E, Bishop DC, Gee TI. Effect of plyometric training on handspring vault performance and functional power in youth female gymnasts. PLoS ONE. 2016;11(2):e0148790. https://doi. org/10.1371/journal.pone.0148790.

12. Hammami R, Chaouachi A, Makhlouf I, Granacher U, Behm D. Associations between balance and muscle strength, power performance in male youth athletes of different maturity status. Pediatr Exerc Sci. 2016;28(4):521-34.

13. Hopkins W, Marshall S, Batterham A, Hanin J. Progressive statistics for studies in sports medicine and exercise science. Med Sci Sports Exerc. 2009;41(1):3-12.

14. Khamis H, Roche A. Predicting adult stature without using skeletal age - the Khamis-Roche method. Pediatrics. 1994;94(4 Pt 1):504-7.

15. Kirby T, McBride J, Haines T, Dayne A. Relative net vertical impulse determines jumping performance. J Appl Biomech. 2011;27(3):207.

16. Komi P, Bosco C. Utilization of stored elastic energy in leg extensor muscles by men and women. Med Sci Sport. 1978;10(4):261-5.

17. Kubo K, Teshima T, Ikebukuro T, Hirose N, Tsunoda N. Tendon properties and muscle architecture for knee extensors and plantar flexors in boys and men. Clin Biomech. 2014;29(5):506-11.

18. Lazaridis S, Bassa E, Patikas D, Giakas G, Gollhofer A, Kotzamanidis C. Neuromuscular differences between pre- pubescents boys and adult men during drop jump. Eur J Appl Physiol. 2010;110(1):67-766.

19. Lloyd R, Cronin J. Plyometric development in youth. In: Lloyd RS, Oliver JL, editors. Strength and conditioning for young athletes science and application. 1st ed. Oxon: Routledge; 2014. p. 100.

20. Lloyd R, Meyers R, Oliver J. The natural development and trainability of plyometric ability during childhood. J Strength Cond Res. 2011;33(2):23-32.

21. Lloyd R, Oliver J, Faigenbaum A, Myer G, De Ste CM. Chronological age vs. biological maturation- implications for exercise programming in youth. J Strength Cond Res. 2014;28(5):1454-64.

22. Lloyd R, Oliver J, Hughes M, Williams C. The influence of chronological age on periods of accelerated adaptation of stretch-shortening cycle performance in pre and postpubescent boys. J Strength Cond Res. 2011;25(7):1889-977. https://doi. org/10.1519/JSC.0b013e3181e7faa8.

23. Lloyd R, Oliver J, Hughes M, Williams C. Specificity of test selection for the appropriate assessment of different measures of stretch-shortening cycle function in children. J Sports Med Phys Fitness. 2011;51(4):595-602.

24. Lloyd R, Oliver J, Hughes M, Williams V. Reliability and validity of field-based measures of leg stiffness and reactive strength index in youths. J Sports Sci. 2009;27(14):1565-73. https://doi. org/10.1080/02640410903311572. 
25. Lloyd R, Oliver J, Radnor J, Rhodes B, Faigenbaum A, Myer G. Relationships between functional movement screen scores, maturation and physical performance in young soccer players. J Sports Sci. 2015;33(1):11-9. https://doi.org/10.1080/02640 414.2014.918642.

26. Lloyd R, Radnor J, De Ste CM, Cronin J, Oliver J. Changes in sprint and jump performances after traditional, plyometric, and combined resistance training in male youth pre- and post-peak height velocity. J Strength Cond Res. 2015;30(5):1239-47.

27. Malina R, Bouche R, Bar-Or O. Growth, maturation, and physical activity. Champaign: Human Kinetics; 2004.

28. Marina M, Jemni M. Plyometric training performance in eliteoriented prepubertal female gymnasts. J Strength Cond Res. 2014;28(4):1015-25. https://doi.org/10.1519/JSC.0000000000 000247.

29. Marina M, Jemni M, Rodríguez F. Jumping performance profile of male and female gymnasts. J Sports Med Phys Fitness. 2013;53(4):378-86.

30. Marina M, Torrado P. Does gymnastics practice improve vertical jump reliability from the age of 8 to 10 years? J Sports Sci. 2013;31(11):1177-86. https://doi.org/10.1080/02640 414.2013.771816.

31. Marina M, Rodríguez FA. Usefulness and metabolic implications of a $60 \mathrm{sec}$ repeated jumps test as a predictor of acrobatic jumping performance in gymnasts. Biol Sport. 2013;30(1):9-15.

32. Meyers RW, Oliver JL, Hughes MG, Lloyd RS, Cronin JB. Influence of age, maturity, and body size on the spatiotemporal determinants of maximal sprint speed in boys. J Strength Cond Res. 2017;31(4):1009-166. https://doi.org/10.1519/JSC.0000000000 001310.

33. Michel M, Monem J, Ferran R. A two-season longitudinal followup study of jumps with added weights and counter movement jumps in well-trained pre-pubertal female gymnasts. J Sports Med Phys Fitness. 2014;54(6):731-41.

34. Moeskops S, Oliver J, Read P, Cronin J, Myer G, Haff G, Lloyd R. Within- and between-reliability of the isometric mid-thigh pull in young female athletes. J Strength Cond Res. 2018;32(7):1892-901.

35. Moeskops S, Oliver J, Read P, Cronin J, Myer G, Haff G, Lloyd $\mathrm{R}$. The influence of biological maturity and competitive level on isometric force-time curve variables and vaulting performance in young female gymnasts. J Strength Cond. 2020. (Epub ahead of print).

36. Moeskops S, Oliver J, Read P, Cronin J, Myer G, Lloyd R. The physiological demands of youth artistic gymnastics; applications to strength and conditioning. J Strength Cond. 2019;41(1):1-13.

37. Moeskops S, Read P, Oliver J, Lloyd R. Individual responses to an 8-week neuromuscular training intervention in trained prepubescent female artistic gymnasts. Sports. 2018;6(4):128.

38. Morin JB, Jimenez-Reyes P, Brughelli M, Samozino P. When jump height is not a good indicator of lower limb maximal power output: theoretical demonstration, experimental evidence and practical solutions. Sports Med. 2019;49(7):999-1006. https:// doi.org/10.1007/s40279-019-01073-1.
39. Motoshima Y, Kitagawa J, Maeda A. The relationship between the mechanical parameters in the take-off of a vault and the drop jump ability. Sci Gymn J. 2015;7(3):37-45.

40. O'Brien T, Reeves N, Baltzopoulos V, Jones D, Maganaris C. Muscle-tendon structure and dimensions in adults and children. J Anat. 2010;216(5):631-42. https://doi.org/10.111 $1 / \mathrm{j} .1469-7580.2010 .01218 . x$.

41. O'Donoghue P. Statistics for sport and exercise studies: an introduction, vol. 1. London: Routledge; 2012.

42. Oliver J, Smith P. Neural control of leg stiffness during hopping in boys and men. J Electromyogr Kinesiol. 2010;20(5):973-9.

43. Owens N, Watkins J, Kilduff L, Bevan H, Bennett M. Development of a criterion method to determine peak mechanical power output in a countermovement jump. J Strength Cond Res. 2014;28(6):1552-8.

44. Pauda D, Carcia C, Arnold B, Granata K. Gender differences in leg stiffness and stiffness recruitment strategy during two-legged hopping. J Motor Behav. 2005;37(2):111-25.

45. Pedley J, Lloyd R, Read P, Moore I, Oliver J. Drop jump: a technical model for scientific application. J Strength Cond. 2017;39(5):36-44.

46. Pion J, Lenoir M, Vandorpe B, Segers V. Talent in female gymnastics: a survival analysis based upon performance characteristics. Int J Sports Med. 2015;36(11):935-40. https://doi. org/10.1055/s-0035-1548887.

47. Polishchuk T, Mosakowska M. The balance and jumping ability of artistic gymnastics competitors of different ages. Med Sport Press. 2007;13(1):100-3.

48. Radnor J, Oliver J, Waugh C, Myer G, Moore I, Lloyd R. The influence of growth and maturation on stretch-shortening cycle function in youth. Sports Med. 2018;48(1):57-71. https://doi. org/10.1007/s40279-017-0785-0.

49. Sleeper M, Kenyon L, Casey E. Measuring fitness in female gymnasts: The gymnastics functional measuring tool. Int J Sports Phys Ther. 2012;7(2):124-38.

50. Suchomel T, Sands W, McNeal J. Comparison of static, countermovement, and drop jumps of the upper and lower extremities in US Junior national team male gymnasts. Sci Gymn J. 2016;8(1):15-30.

51. Turner A, Comfort P, McMahon J, Bishop C, Chavda S, Read P, Mundy P, Lake J. Developing powerful athletes, Part 1: mechanical underpinnings. J Strength Cond. 2020;42(3):30-9.

52. Turner A, Jeffreys I. The stretch-shortening cycle: proposed mechanisms and methods for enhancement. J Strength Cond. 2010;32(4):87-99.

53. Vandorpe B, Vandendriessche JB, Vaeyens R, Pion J, Lefevre J, Philippaerts RM, Lenoir M. The value of a non-sportspecific motor test battery in predicting performance in young female gymnasts. J Sports Sci. 2012;30(5):497-505. https://doi. org/10.1080/02640414.2012.654399.

54. Winter D. Biomechanics and motor control of human movement. 4th ed. Hoboken: Wiley; 2009. 